\title{
STUDY OF THE INCIDENCE AND EFFECT OF MECONIUM STAINED AMNIOTIC FLUID ON FETAL MORBIDITY AND MORTALITY OF NEONATES BORN
}

\author{
Obstetrics \& Gynaecology
}

Dr. Kajal Kunwar* MS Obs \& Gynae, Patna, Bihar. *Corresponding Author

\section{Dr. Shanti HK Singh}

MS Obs \& Gynae, Patna, Bihar.

\section{ABSTRACT}

AIMS AND OBJECTIVES: Meconium stained amniotic fluid was considered a sign of fetal distress and associated with poor fetal outcome, but others considered physiological phenomena to be meconium passage through the fetus and create environmental threats to the fetus before birth. Such magnitude of different opinions was the object behind taking up this study and the aim was to find out the incidence and effect of meconium in terms of morbidity and mortality. MATERIALAND METHODS: The present study was undertaken to evaluate the significance of MSAF and its fetal outcome in parturients admitted to a tertiary care hospital between June 2012 to June 2014. Detection of MSAF during delivery and follow-up of mother and baby during hospital stay was done. A total number of 100 cases were studied in each group as a prospective study. RESULTS: The total numbers of deliveries during the study period were 850 of which 100 cases had meconium staining of AF (11.6\%). Thin meconium staining was seen in 37 cases $(4.35 \%)$ and Thick meconium was seen in 63 cases $(7.41 \%)$. The major neonatal complication was birth asphyxia in MSG $(19 \%)$ which was more in thick MSG (14\%). Neonatal morbidity was more in the newborn with the thick meconium group (36.5\%) compared to the thin meconium-stained group (29.7\%). Early neonatal mortality was $100 \%$ associated with thick MSG. Early neonatal death was 2 in thick MSG and it was due to MAS. Stillbirth was $100 \%$ associated with thick MSG and it was 4 . Whereas stillbirth in the control group was 1 . Perinatal mortality was $6 \%$ in MSG that was associated with thick MSG. In the control group, it was $1 \%$. Consistency of meconium has a direct bearing on the fetal outcome. In the thick meconium-stained group, Neonatal morbidity was (in our study group) 23 out of 63 cases. Stillbirth was 4 ; early neonatal death was 2 out of 63 cases. Whereas in thin MSG neonatal morbidity was 11 out of 37 cases. No stillbirth or neonatal death occurred in thin MSG. CONCLUSION: Immediate airway management, need for suction, and intubation should be guided by the state of the newborn rather than the presence of meconium. Timely diagnosis and management of amniotic fluid stained with meconium can enhance the fetal outcome. The authors of the current study conclude that MSAF adversely affects the fetal outcome mainly by thick meconium.

\section{KEYWORDS}

Meconium Stained Amniotic Fluid, Birth Asphyxia, Morbidity, Mortality

\section{INTRODUCTION}

Meconium, the gastrointestinal excreta of the fetus was named by Aristotle. The word meconium is derived from the Greek word 'Meconium Arion' which means like opium or poppy like substances caused a sleeping state of the fetus in mother's womb.

Amniotic Fluid Meconium Staining (MSAF) is a relatively common issue that occurs in $8-15 \%$ of all deliveries. MSAF is considered a harbinger of fetal compromise because of its direct correlation with fetal distress and increased likelihood of aspiration of meconium with resultant deleterious effects on the neonatal lung.

The first intestinal discharge from a newborn is Meconium, which is a viscous, dark green substance composed of intestinal epithelial cells, lanugo, mucus, and intestinal secretions, such as bile. The 3 main solid components of Meconium are intestinal secretions, mucosal cells, and solid elements of swallowed amniotic fluid. The main liquid constituent is water, which makes up $85-95$ percent of meconium. It can cause intrauterine pain to pass into the amniotic fluid. Factors that promote the passage in-utero include placental insufficiency, maternal hypertension, pre-ecclampsia, oligohydramnios, and maternal drug abuse, especially of tobacco and cocaine. Meconium Stained Amniotic Fluid may be aspirated during labor and delivery, causing neonatal respiratory distress. Because Meconium is rarely found in the amniotic fluid before 34 weeks' gestation, Meconium Aspiration chiefly affects infants at term and post-term. Ostara found that $78 \%$ of MSAF occurred between 38 and 42 weeks of gestation. MAS develop in 5-10 $\%$ of the babies born through MSAF. Meconium Aspiration Syndrome (MAS) is a disease with complex pathophysiology \& potential for mortality and considerable morbidity. Despite the recent advances in neonatal care, at least $5 \%$ of MAS babies admitted to the neonatal intensive care unit (NICU) have been reported to die. In the neonatal phase, five percent of MAS babies need oxygen. Complications acquired by babies, including persistent fetal circulation.

Preventing maternal and fetal morbidity and mortality is the main objective of obstetric care. To reduce fetal morbidity and mortality early identification of markers of fetal distress is important. The classical sign or markers of fetal hypoxia are loss of or decreased fetal movements, variations in fetal heart rate pattern, presence of meconium in $\mathrm{AF}$, presence of fetal molding, and decrease in fetal scalp blood $\mathrm{pH}$
Since there are no adverse outcomes for all fetuses with meconium passage in labor, it is necessary to discern those destined to develop fetal distress promptly and intervene to avoid MAS and sequelae accordingly.

Hence the present study of "Incidence and Effect of meconium-stained amniotic fluid on fetal morbidity and mortality" has been undertaken to note the frequency of meconium staining of $\mathrm{AF}$ in obstetrical practice, to whether all meconium-stained cases result in asphyxiated birth, to whether the amount of meconium discharge into the AF has any relation with fetal outcome.

\section{MATERIALS AND METHODS}

The present study was undertaken to evaluate the significance of MSAF and its fetal outcome in patients admitted to a tertiary care hospital between June 2012 to June 2014. Inclusion criteria were Term labor (>37 completed weeks), Cephalic presentation, Live singleton pregnancy and Pregnancy without any congenital malformation.

Exclusion criteria were Pre-term labor ( $<37$ completed weeks), Antepartum hemorrhage, Breech presentation, Transverse lie, Multiple pregnancies, Pregnancy with congenital malformations and Intrauterine death

Control cases were selected simultaneously with the study case to avoid any other factors like seasonal variation, overcrowding in the ward, etc., influencing the outcome. Mothers in labor were studied when meconium was found at the time of rupture of membrane or when clear $\mathrm{AF}$ turned meconium-stained during labor

Following the selection of cases a detailed history regarding age, gravida, and parity, past obstetrical history, menstrual history, socioeconomic status, history of present pregnancy, history of medical and surgical disorders were noted from the patients' antenatal records. Detection of MSAF during delivery and follow-up of mother and baby during hospital stay was done. A total number of 100 cases were studied in each group as a prospective study.

\section{RESULTS}

Total numbers of deliveries during the study period were 850 of which 100 cases had meconium staining of AF, which constituted $11.76 \%$ of total deliveries. Out of 850 deliveries, 100 cases of MSAF were studied 
for 17 months. The incidence of meconium staining of AF in this study is limited to up to 100 cases. $4.35 \%$ of deliveries had thin meconium staining and $7.41 \%$ had thick meconium staining (Figure 1).

\section{Frequency and Type of Meconium Staining of AF of Total Deliveries}

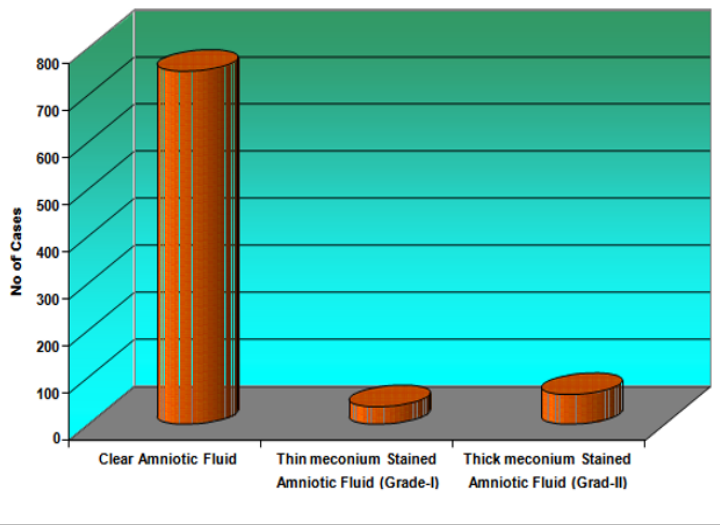

Figure 1: Frequency and type of meconium staining of AF of total deliveries

Among the cases of meconium staining of AF, Thin meconium constituted $37 \%$ of cases and thick meconium in $63 \%$ cases. Maximum number of cases was thick meconium stained. Incidence of meconium stained AF has been shown in different age groups compared to the control group with clear AF. In the study group, the maximum numbers of cases $(50 \%)$ were in the age group of $21-25$ years and the mean age group was 22.53 years. In the control group also the maximum number of cases (48\%), were in the same age group and the mean age was 22.54 years. Cases were selected with age-matched in the control group with meconium stained group. So the percentage of cases in the different age groups was almost the same in control and meconium stained cases.

Neonatal morbidity was highest in thick meconium a group baby, which was about $36.5 \%$ whereas in the control group it was less, was about $19 \%$. Total morbidity was about (34\%) 2 times more in meconium stained group than that of in control group (19\%).

The major complications was birth asphyxia (about 1.5 times more) than that of MAS. Complications were more common in the thick group. Out of total deliveries (850) of the study period, MAS developed in $1.4 \%$ of babies, and out of 100 MSAF cases, 12 babies $(12 \%)$ developed MAS.

Table 1 shows that in the study Group 4 stillbirth occurred in contrast to the control group it was 1 i.e. stillbirth 4 times more in meconium stained group. There was no first-week neonatal death in the control group. Perinatal death was 6 times more in the study group than the control group. Out of total deliveries (850) of the study period, stillbirth was $0.5 \%$ in MSG whereas in the control group it was $0.12 \%$.

Table 1: Shows incidence of perinatal death in relation to different grade of meconium in study group compared to control group.

\begin{tabular}{|c|c|c|c|c|c|c|c|c|}
\hline \multirow{2}{*}{\multicolumn{2}{|c|}{ Group }} & \multirow[t]{2}{*}{$\begin{array}{l}\text { No. } \\
\text { of } \\
\text { Cases }\end{array}$} & \multicolumn{2}{|c|}{ Still birth } & \multicolumn{2}{|c|}{$\begin{array}{l}\text { 1st week } \\
\text { Neonatal } \\
\text { death }\end{array}$} & \multicolumn{2}{|c|}{$\begin{array}{l}\text { Perinatal } \\
\text { death }\end{array}$} \\
\hline & & & $\begin{array}{l}\text { No. of } \\
\text { Cases }\end{array}$ & $\%$ & $\begin{array}{l}\text { No. of } \\
\text { Cases }\end{array}$ & $\%$ & $\begin{array}{l}\text { No. of } \\
\text { Cases }\end{array}$ & $\%$ \\
\hline \multirow{3}{*}{$\begin{array}{l}\text { Study } \\
\text { Group } \\
(\mathbf{N}=\mathbf{1 0 0})\end{array}$} & $\begin{array}{l}\text { Thin Group } \\
(\mathrm{n} 1=37)\end{array}$ & 37 & 0 & 0 & 0 & 0 & 0 & 0 \\
\hline & $\begin{array}{l}\text { Thick Group } \\
(\mathrm{n} 2=63)\end{array}$ & 63 & 4 & 6.34 & 2 & 3.17 & 6 & 9.5 \\
\hline & Total & 100 & 4 & 4 & 2 & 2 & 6 & 6 \\
\hline \multicolumn{2}{|c|}{ Control Group $(\mathbf{N}=\mathbf{1 0 0}$} & 100 & 1 & 1 & 0 & 0 & 1 & 1 \\
\hline
\end{tabular}

Table 2 shows that in the meconium-stained group all death was due to MAS. There was no first-week neonatal death in the control group whereas it was $2 \%$ in MSG. Out of a total of 12 MAS babies 2 died in the first-week neonatal period - it was $16.66 \%$ of total MAS babies.
Table 2: Shows the cause and incidence of first week neonatal death

\begin{tabular}{|l|l|l|l|l|l|l|}
\hline Causes & \multicolumn{3}{|l|}{ Study Group } & \multicolumn{2}{l|}{ Control Group } \\
\cline { 2 - 7 } & $\begin{array}{l}\text { No. of } \\
\text { Cases }\end{array}$ & $\begin{array}{l}\text { No. of } \\
\text { death }\end{array}$ & $\begin{array}{l}\text { No. of } \\
\text { Cases }\end{array}$ & $\begin{array}{l}\text { No. of } \\
\text { death }\end{array}$ & $\%$ \\
\hline MAS & 12 & 2 & 16.66 & - & - & - \\
\hline Pneumonia & - & - & - & - & - & - \\
\hline Asphyxia Neonatorum & 19 & - & - & 11 & - & - \\
\hline Septicemia & - & - & - & 3 & - & - \\
\hline $\begin{array}{l}\text { Hypoxic ischaemic } \\
\text { encephalopathy }\end{array}$ & - & - & - & - & - & - \\
\hline Physiological Jaundice & 3 & - & - & 5 & - & - \\
\hline
\end{tabular}

No. of babies of early neonatal death in MSG (study group) - 2 and in Control group -0

Figure 2 shows that neonatal morbidity in the Study Group was more about $34 \%$ in comparison to $19 \%$ in the control group. In the study Group, stillbirth was $4 \%$ which was about 4 times the control group (1\%). The thick meconium-stained group was more complicated than that of the thin meconium-stained group outcome. Early neonatal death was $2 \%$ in the study group, it was nil in the control group. Perinatal morbidity was $38 \%$ and perinatal mortality was $6 \%$ in the study group, those were in the control group $20 \%$ and $1 \%$ respectively.

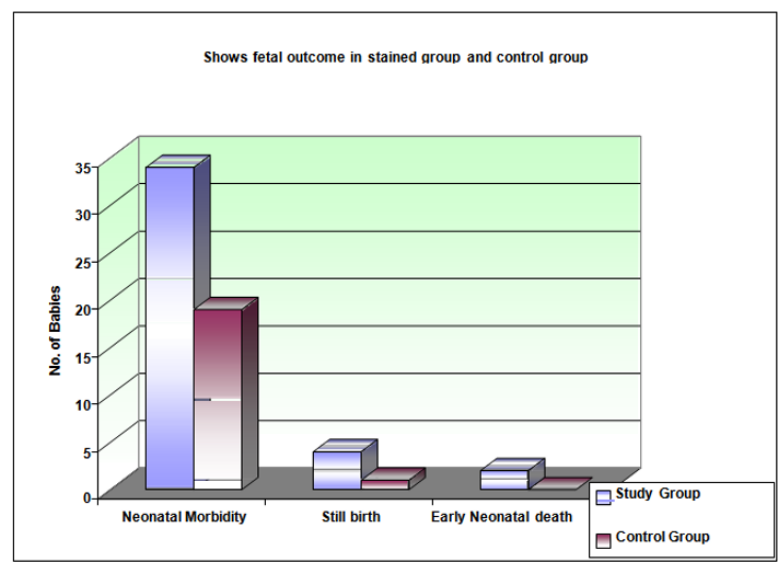

Figure 2: Shows fetal outcome in meconium stained group and control group

\section{DISCUSSION}

SAF has been implicated as a factor influencing the fetal wellbeing during the intrapartum and postpartum periods. Even for old-age midwives and obstetricians, the presence of meconium in AF in the cephalic presentation was of great concern. It was insignificant in breech presentation as it was thought to be expressed by mechanical compression of the soft abdomen of the fetus. Passage of meconium was once thought to be a sure sign of fetal death in utero but later it was realized to be a sign of fetal hypoxia, not fetal death. Even modern obstetricians are well aware of this reality, and in the light of meconium in AF during childbirth, which needs close vigilance of the fetal wellbeing, they cannot be indifferent.

Of the 850 deliveries conducted in our hospital during this period 100 cases were included in our study which fulfilled the inclusion criteria. Of the 100 cases, 37 had thin MSAF (MSAF) and 63 had thick MSAF observed at the time of membrane spontaneous or artificial rupture of membranes.

As recorded from time to time by various workers, the incidence of MSAF in labour varies widely. In the present study the incidence was $11.76 \%$. Goud and Krishna (1) in their study found incidence of MSAF as 9.8\%, Arun Nayek (2) had $14 \%$ and Hari Bhaskar (3) found incidence as $11.2 \%$.

From the above list, it is evident that the incidence varies from 9.8 to $14 \%$. In the present study among the MSAF cases, the highest incidence was thick MSAF. Of the total deliveries of our study period, $4.35 \%$ of cases had thin MSAF and $7.41 \%$ of cases had thick MSAF.

In the present study neonatal morbidity was 34\% in the study group, whereas it was in the control group $19.19 \%$. Morbidity was more in the 
thick group $36.5 \%$ than that of in thin group $29.7 \%$. Arun Nayek (2) in their study found thin meconium in $5.58 \%$ and thick meconium in $8.18 \%$ of sample population. Similarly another study by Debdas (4) found incidence of meconium as $10 \%$.

Neonatal morbidity in our study is significantly associated with MSAF $(\mathrm{P}<0.02)$. In our present study perinatal death was $6 \%$ in the study group. Goud and Krishna (1), Debdas (4), Hellman (5) and Narang (6) in their series found perinatal mortality ranged from $3 \%$ to $7.7 \%$. They had similar observations as compared to the present study. In the present study $100 \%$, perinatal mortality was in the thick meconium group. Debdas (4) opined that in the group with thin meconium the babies are not generally depressed at birth and do not have any higher perinatal mortality rate in comparison to those with the clear group.

Perinatal death more in MSG in our study, it is not statistically significant $(\mathrm{P}>0.05)$.

In the present series 1 st-week neonatal death was $2 \%$, it is $100 \%$ due to MAS. So, meconium was responsible for $100 \%$ of neonatal death in our study. Other workers like Arun Nayek (2) observed 3.42\% neonatal death.

First week neonatal death is common in MSG in our study but it is not statistically significant.

In our study mortality and morbidity in MSAF is comparable to other studies as observed by Usha (2004), where morbidity was $32.6 \%$ and mortality $6 \%$.In our study it was $34 \%$ and $6 \%$ respectively .

\section{CONCLUSION}

The overall incidence of meconium staining of AF during labor was in the present study $11.6 \%$. The incidence of meconium staining was much greater in postdated pregnancy and oxytocin-induced labor. Birth asphyxia was more common when the AF was meconium stained and severity was directly proportional to the degree of thickness of MSAF. Immediate airway management, need for suction and intubation should be guided by the state of the newborn, rather than the presence of meconium. To the obstetrician, the Meconium passage remains an enigma. However, as shown in the study, thick or thin meconium is indicative of fetal distress. If modern management is based on the perception of the meconium passage's underlying pathophysiology, then the harmful impact of meconium will be decreased.

Immediate airway management, need for suction and intubation should be guided by the state of the newborn rather than the presence of meconium. Timely diagnosis and management of meconium-stained amniotic fluid may improve fetal outcome. From the present study, the authors conclude that MSAF adversely affects fetal outcome mostly by thick meconium.

\section{REFERENCES}

. Goud P and Krishna U. Significance of meconium staining of amniotic fluid in labor. Journal of Obstetrics and Gynaecology of India 1989;39:523-526.

2. Arun H Nayek, Asha R Dalal. Meconium staining of amniotic fluid -significance and fetal outcome. Journal of Obstetrics and Gynaecology 2001; 41:480-483.

3. Hari Bhaskar S, Karthikeyan G, Vishnu Bhat B, Bhatia BD. Antenatal risk factors and neonatal outcome in meconium aspiration syndrome.Indian J. Maternal and Child Health 2007; 8(1): 9-12.

4. Debdas AK, KaurT. Meconium stained liquor-Reappraisal.Journal of Obstetrics and Gynaecology of India 1981;31:924-929.

5. Hellman LM, Schiffer MA, Kohl SG and Tolles WE. Studies in fetal well being: Variations in fetal heart rate. Am. J. Obstet. Gynecol. 1958; 76: 998-1012.

6. Narang A, Nair PMC, Bhakoo ON, Vashist K. Management of meconium sainted amniotic fluid: A team approach, Indian Pediatr 1993; 30:9-13.

7. Usha Sharma, Kamala Gokhroo, Mamta Sharma. Perinatal outcome in meconiumstained amniotic fluid. As J Obstet Gynecol Practice 2004; 8(4): 37-40. 